\title{
Proteomic identification of potential target proteins regulated by an ASK1-mediated proteolysis pathway
}

\author{
Xiaofeng Wang ${ }^{1}$, Weimin $\mathrm{Ni}^{2}$, Xiaochun $\mathrm{Ge}^{1}$, Jiejin Zhang ${ }^{1}$, Hong $\mathrm{Ma}^{2}$, Kaiming $\mathrm{Cao}^{1}$ \\ ${ }^{1}$ State Key Laboratory of Genetic Engineering, Department of Biochemistry and Molecular Biology, School of Life Sciences, Fudan \\ University, Shanghai, 200433, China; ${ }^{2}$ Department of Biology and the Huck Institutes of the Life Sciences, the Pennsylvania State \\ University, University Park, Pennsylvania 16802, USA
}

The ASK1 (ARABIDOPSIS SKP1-LIKE) protein is a critical component of the SCF (Skp1-Cullin-F box protein) ubiquitin ligase complexes that recruit target proteins for degradation by the $26 \mathrm{~S}$ proteosome. To investigate proteins that are affected by the ASK1-mediated proteolysis pathway in Arabidopsis flowers, we compared the proteomes of the Arabidopsis wild type and ask1 mutant flower buds using two-dimensional electrophoresis (2-DE). Ten protein spots with higher or lower abundance in the askl mutant flowers compared to wild type flowers were excised and subjected to further mass spectrometry (MS) analysis. The results showed that they were proteins involved in photomorphogenesis, circadian oscillation, post-translation process, stress-responses and cell expansion or elongation, suggesting that those processes were affected in the askl mutant. The transcript levels of these genes were also compared based on the Affymetrix gene chip microarray data. No significant difference was observed for most of the genes, suggesting that the proteins with elevated levels of accumulation in the ask1 mutant could be candidate targets regulated by an ASK1-mediated proteolysis pathway. These results help to elucidate the pleiotropic functions of ASK1 in Arabidopsis developmental processes and also demonstrate the importance and necessity of studying protein levels with respect to gene functions.

Cell Research (2006) 16:489-498. doi:10.1038/sj.cr.7310060; published online 15 May 2006

Keywords: ASK1, two-dimensional polyacrylamide gel electrophoresis, mass spectrometry, proteolysis

\section{Introduction}

Floral development is one of the most complicated developmental processes in plants that involve a series of genes interacting with each other to regulate the entire process. One of the more recently identified regulatory genes of flower development is ASK1 (Arabidopsis SKP1-like 1), which is required for normal floral organ development and homeotic gene expression [1-3]. SKP1 (S-phase kinase-

Correspondence: Xiaochun $\mathrm{Ge}^{1}$, Hong $\mathrm{Ma}^{2}$

${ }^{1}$ Tel: +86-21-65643672; Fax: +86-21-65650149;

E-mail: xcge@fudan.edu.cn

${ }^{2}$ Tel: +01-814-863-6414; Fax: +01-814-863-1357;

E-mail: hxm16@psu.edu

Received 13 Feb 2006; revised 14 Mar 2006; accepted 21 Mar 2006; pub-

lished online 15 May 2006 associated protein) was originally identified in human and yeast as an essential component of the ubiquitin-ligase complex called SCF (SKP1, Cullin/Cdc53p and F-box containing protein) [4-6]. The SCF complex targets several specific proteins in mitotic cell cycle for proteolysis $[6,7]$. In the Arabidopsis genome, 21 ASK genes are found while the functions of most of them remain unknown $[8,9]$.

The first mutant in an $A S K$ gene, ask1-1, was isolated following transposon insertional mutagenesis, allowing genetic analysis of $A S K 1$ function [1]. This mutant is defective in both vegetative and reproductive development, including smaller stature than normal, reduced number and size of petals and stamens, and reduced stamen filament length $[1,2]$. Further microscopic examination revealed that it is defective in male meiosis, exhibiting an uneven distribution of chromosomes and a failure in separation of some homologous chromosomes during male anaphase I, thus 
resulting in male-sterility. ASK1 may regulate homologous chromosome separation by promoting the degradation or removal of a protein that is required for the association of two homologous chromosomes [1].

In addition to a role in meiosis, ASK1 was also shown to interact with UFO (UNUSAL FLORAL ORGANS) protein to regulate $\mathrm{B}$ function gene expression during the flower morphogenesis process [3, 10]. Moreover, ASK1 was reported to interact with several other F-box proteins to regulate plant growth and development, including TIR1 in auxin response [11, 12], COI1 in jasmonic acid-regulated defense response [13, 14], EID1 and COP9 in light signaling $[15,16]$, and ZTL and FKF1 in the control of the circadian clock $[17,18]$. ASK1 is strongly and widely expressed in plants [8], implying an important function in various plant tissues. In particular, $A S K 1$ is important for embryo and seedling development [19].

Many functionally important proteins in eukaryotes are short-lived and should be rapidly removed when not needed. One way to attain this goal is degradation through the ubiquitin-dependent proteolysis pathway [9]. Ubiquitin is a 76-residue protein that can be covalently attached to target proteins through an enzymatic conjugation cascade involving three enzymes: the ubiquitin-activating enzyme (E1), ubiquitin-conjugating enzyme (E2) and ubiquitin ligase (E3) $[7,10,14]$. E1 and E2 are non-specific, whereas E3 confers substrate specificity and thus attracts more attention [20]. In Arabidopsis, the ASK1 protein is a critical component of SCF E3 complexes that also contains Cullin (CDC53 in yeast), Rbx1/Hrt1/Roc1 and one of F-box containing proteins $[5,6,7]$. Through the catalysis by E1, E2 and E3, target proteins are polyubiquitinated and rapidly presented to the $26 \mathrm{~S}$ proteasome for degradation. In both auxin and GA responses, degradation of transcriptional repressors of hormone-inducible genes is mediated by SCF complexes containing specific F-box proteins $[12,21]$.

In recent years, ubiquitin-dependent proteolysis has been shown to be important in the regulation of various cellular processes, including cell cycle progression, signal transduction, transcription, DNA repairing, and protein quality control [6-11]. Although multiple F-box proteins interacting with ASK1 have been reported, little is known about its down-stream substrates that are recruited by the SCF E3 complexes. To investigate the potential protein targets degraded by the ASK1-mediated proteolysis pathway and also the roles of ASK1 in the Arabidopsis floral development, we compared the proteomes between Arabidopsis wild type and the ask1 mutant flower buds using the tool of two-dimensional electrophoresis. More than 3000 protein spots were resolved in the 2-DE gels. 10 protein spots showing abundance difference between the wild type and the ask1 mutant were isolated for mass spectrometry analysis.
Seven of them had much higher levels in the ask1 mutant than in wild type. Their transcript levels in wild type and in the ask 1 mutant were also compared based on the gene chip microarray data. For most of the proteins identified in our experiments, there is no significant transcript difference between the wild type and the ask 1 mutant, which is consistent with the idea that the levels of these proteins are regulated in the post-transcription manner and they might be target proteins degraded by an ASK1 mediated ubiquitin-dependent proteolysis pathway. These results contributed to the understanding of the pleiotropic functions of the $A S K 1$ gene in reproductive development, and also illustrate the importance of determining protein levels when studying gene functions.

\section{Materials and methods}

\section{Plant materials and growth conditions}

The Arabidopsis wild type and the ask1 mutant plants used in this study are of the Landsberg erecta (Ler) ecotype. All plants were grown at $23^{\circ} \mathrm{C}$ under a long-day condition $(16 \mathrm{~h}$ light and $8 \mathrm{~h}$ dark). The closed flower buds up to stage 11 to 12 were harvested and stored at $-70{ }^{\circ} \mathrm{C}$ until use. Proteomes from at least three independently grown populations of wild type and the askl mutant were compared.

\section{Protein preparation and quantification}

$20 \mathrm{~g}$ flower buds were ground in a mortar with liquid nitrogen, and then suspended in a buffer $(10 \% \mathrm{w} / \mathrm{v}$ trichloroacetic acid in acetone and $0.07 \% \mathrm{v} / \mathrm{v} 2$-mercaptoethanol). After incubation at $-20{ }^{\circ} \mathrm{C}$ for $45 \mathrm{~min}$, the samples were centrifuged at the $35000 \mathrm{~g}$ for $15 \mathrm{~min}$. The supernatant was discarded and the pellet was washed in cold $\left(-20{ }^{\circ} \mathrm{C}\right)$ acetone containing $0.07 \% \mathrm{v} / \mathrm{v} 2$-mercaptoethanol and $1 \mathrm{mM}$ Phenylmethylsulfonyl Fluoride (PMSF, Sigma, St.Louis, USA), and freeze-dried. $20 \mathrm{mg}$ protein powders were resuspended in $400 \mu \mathrm{l}$ sample solution containing $6 \mathrm{M}$ urea, $2 \mathrm{M}$ thiourea (Sigma, St. Louis, USA), 2\%w/v CHAPS (Amersham Pharmacia Biotech, Buckinghamshire, England ), 2\%w/v sulfobetaine 10 (SB3-10, Sigma, St. Louis, USA), $65 \mathrm{mM}$ dithiothreitol (DTT, Amersham Pharmacia Biotech, Buckinghamshire, England), 0.001\% bromophenol blue (Sigma, St.Louis, USA), and 0.2\% pH 3-10 carrier Biolyte ampholytes (Bio-Rad, Hercules, USA). Occasional stirring is necessary to accelerate the resuspension. After centrifugation to remove the insoluble debris, protein concentration was estimated by the method of Bradford [22].

\section{Two-dimensional gel electrophoresis}

All experimental procedures were carried out as recommended by the manufacturer (Bio-Rad, Hercules, USA) with a few modifications. Equal amounts of wild type and ask1 flower proteins were applied to immobilized $\mathrm{pH}$ gradient (IPG) strips (Bio-Rad, $17 \mathrm{~cm}$, linear $\mathrm{pH}$ 5-8, and linear $\mathrm{pH} 3-10)$. The IPG strips were passively rehydrated with the protein samples overnight, then focused at $10000 \mathrm{~V}$ for a total of $60000 \mathrm{Vh}$ at $18{ }^{\circ} \mathrm{C}$. Following a two-step equilibration $(15$ min incubation in DTT buffer, and another $15 \mathrm{~min}$ in iodoacetamide buffer), each IPG strip was positioned on a $12.5 \%$ polyacrylamide gel. A sample of protein markers (Invitrogen Benchmark ${ }^{\mathrm{TM}}$, New 
York, USA) was loaded besides the IPG strip. Low-melting agrose gel with bromophenol blue was overlaid on the IPG strip to embed the strip. The electrophoresis was run at $70 \mathrm{~mA}$ until the blue dye reached the bottom of the gel. Gels were fixed and stained in Coomassie Brilliant R-250 solution (50\% v/v ethanol, 5\% v/v acetic acid, and $0.2 \% \mathrm{w} / \mathrm{v}$ R-250) overnight, then destained in a solution containing $7 \% \mathrm{v} / \mathrm{v}$ ethanol and $7 \% \mathrm{v} / \mathrm{v}$ acetic acid until the spots on the gels can be clearly visualized. The gels were scanned with a laser scanner (Molecular Image FX, Bio-Rad, Hercules, USA).

\section{Image analysis}

Digitalized gel images were analyzed with PDQuest software (Bio-Rad, Hercules, USA). Spot features of the images were automatically detected with the same setting parameters. The image of the gel with the wild type sample was selected as the template for the master gel. Each image was divided into six sections for the convenience of analysis. By comparison with the molecular weights of the protein markers on the side of the gels and known Swiss-prot 2-DE protein spots on our gels, the $p I$ and $\mathrm{Mw}$ of each excised proteins were estimated. By repeatedly comparing several batches of parallel gels, those protein spots with at least 2-fold difference in abundance calculated by the computer software and also confirmed by naked eyes were selected for further mass spectrometry analysis.

\section{Protein identification by ESI-Q-TOF}

Protein spots were excised and then subjected to alkylation and tryptic digestion. The gel pieces containing proteins were destained three times with a solution containing $25 \mathrm{mM} \mathrm{NH}_{4} \mathrm{HCO}_{3}$ in $50 \%$ acetonitrile, then dried and re-hydrated with a reducing solution (10 mM DTT in $100 \mathrm{mM} \mathrm{NH}_{4} \mathrm{HCO}_{3}$ ) for $1 \mathrm{~h}$ at $56^{\circ} \mathrm{C}$. After cooling to room temperature, the reduced proteins were alkylated in 100 $\mathrm{mM} \mathrm{NH} \mathrm{HCO}_{3}$ with $0.27 \mathrm{mM}$ iodoacetamide for $45 \mathrm{~min}$. The gel pieces were washed with $100 \mathrm{mM} \mathrm{NH}_{4} \mathrm{HCO}_{3}$ and dehydrated with acetonitrile, then re-hydrated in a digestion solution containing 50 $\mathrm{mM} \mathrm{NH}{ }_{4} \mathrm{HCO}_{3}$ and $10 \mathrm{ng} / \mu \mathrm{l}$ of trypsin (Promega, Madison, USA) at $37^{\circ} \mathrm{C}$ overnight. After the trypsin digestion, the gel pieces were further extracted once with $20 \mathrm{mM} \mathrm{NH}_{4} \mathrm{HCO}_{3}$, twice with $5 \%$ formic acid in 50\% acetonitrile. The combined solution were dried and dissolved in $10 \mu \mathrm{l}$ of $2 \%$ acetonitrile, $0.1 \%$ formic acid. Tryptic-digested peptide masses were measured using an ESI-Q-TOF tandem mass spectrometer. The peptide masses were used to search against the Mascot database (http://www.matrixscience.com/cgi/search_form. pl?FORMVER=2\&SEARCH=PMF) and a protein score of more than 50 was considered as a positive identification.

\section{Microarray experiments}

The microarray experiments were performed as described before [23]. Briefly, RNA from young inflorescence (stage 1-9) was isolated from three independent ask 1 inflorescence samples and two wild type inflorescence samples. 15-20 $\mu \mathrm{g}$ of fragmented cRNA was used to hybridize the Arabidopsis $25 \mathrm{~K}$ GeneChip array (Affymetrix, Santa Clara, USA). The scan data were converted to expression levels using the R-Affy package in Bioconductor (version 1.3.25, Irizarry, Gautier, and Bolstad.) The probe-level data were normalized using RNA normalization across arrays together with arrays from the wild type inflorescence and stage 12 flower produced in the lab before [23].

\section{Results and discussion}

To identify proteins that are potentially targeted for degradation by ASK1-containing SCF complexes, protein samples from the wild type and the ask 1 mutant flowers were prepared and separated by 2-DE electrophoresis in

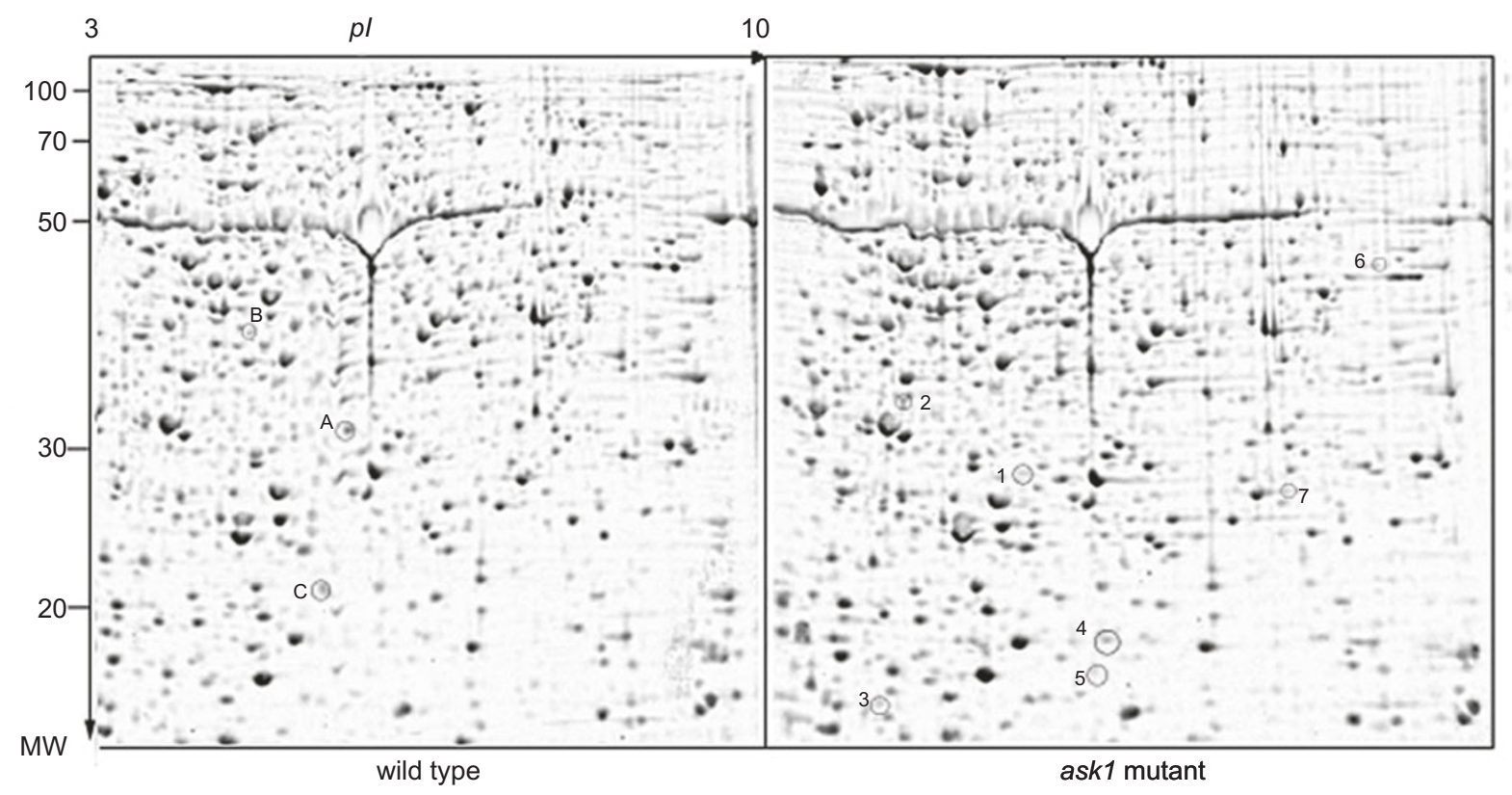

Figure 1 The overview pictures of the 2-D gels obtained for Arabidopsis wild type and ask1 flowers. 

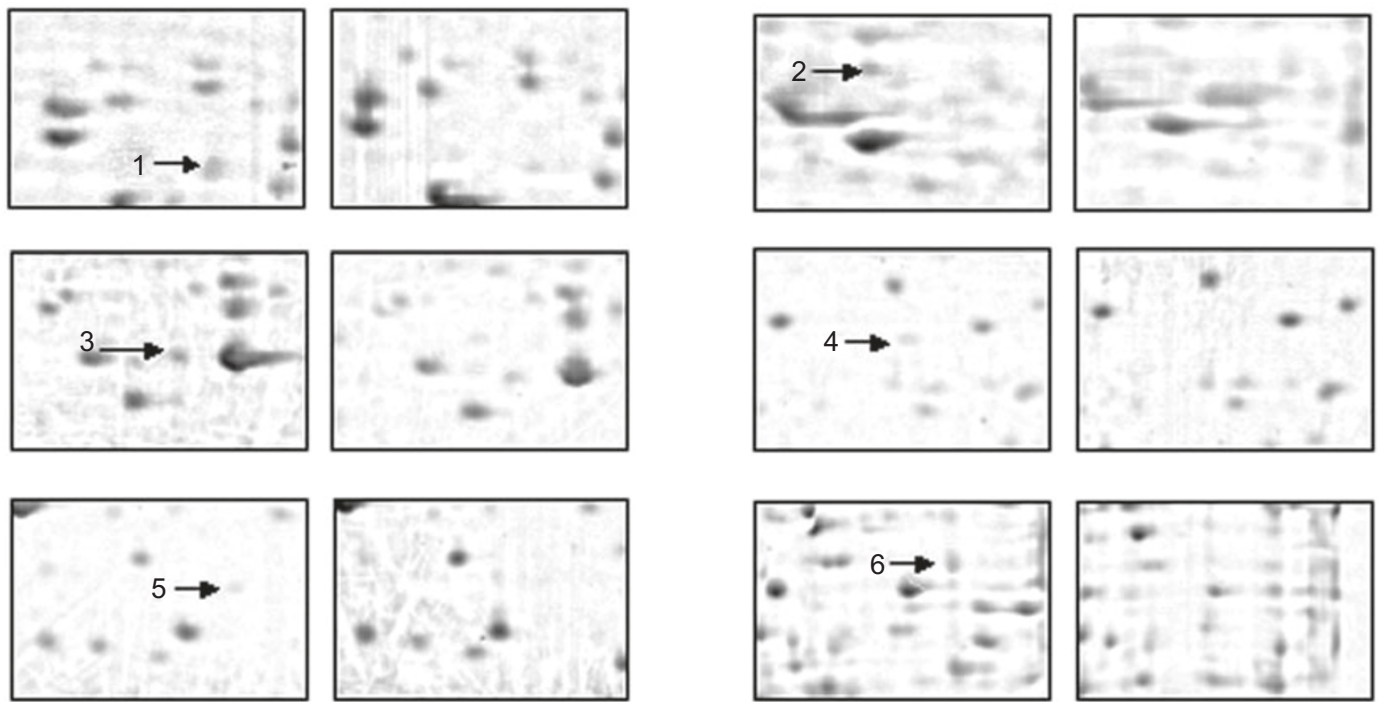

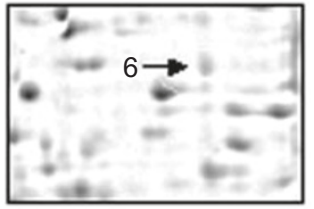

ask1

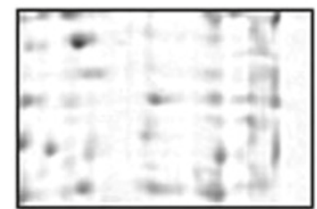

WT

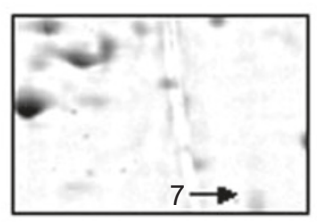

ask1

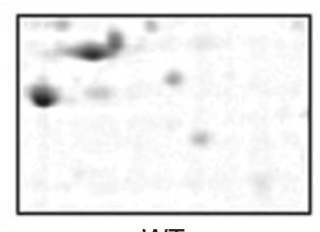

WT

Figure 2 Proteins with higher abundance in the ask1 mutant flowers compared to the wild type flowers. The enlarged images are spot images after digitalization and spot matching. The proteins with different abundance are marked with arrows and numbered. 1, heme oxygenase 1; 2, aldose 1-epimerase family protein; 3, oxygen-evolving complex-related; 4, $17.8 \mathrm{kDa}$ class I heat shock protein; 5 , glycine-rich RNA-binding protein (GRP7), BetVI allergen family protein, nascent polypeptide-associated protein; 6, aspartyl protease family protein and 7 , expressed protein.

parallel. We used the broad range IPG strips of pH3-10 first to obtain the overview pictures of the wild type and ask1 mutant proteomes, and then use pH5-8 IPG strips to ascertain the differences of some protein spots (data not shown). Digital image analysis was performed using the same setting parameters for each gel. The enlarged overview pictures of wild type and the ask1 mutant floral proteomes are shown side by side in Figure 1. The two gels were first auto-matched with the same parameters and then divided into six sections for editing convenience. By repeatedly matching among batches of wild type and the ask 1 mutant gels, those differently expressed protein spots with high reproducibility were sorted and numbered.

Proteins that accumulated at a higher level in the ask1 mutant flowers compared to the wild type

The goal of the present study is to investigate the potential protein targets degraded by the ASK1-mediated proteolysis pathway. The working hypothesis is that, when ASK1 is defective, the protein targets may not be degraded by the $26 \mathrm{~S}$ proteosome and thus remain in the protein extract. By using 2-D gel electrophoresis, we may be able to identify some of those proteins. Based on this hypothesis, the target proteins should exhibit higher abundance in the ask 1 mutant flowers than in the wild type flowers. Comparison of the proteomes of wild type and the askl flowers revealed that seven protein spots (Figure 2) were always more abundant in the ask1 mutant than in the wild type for different batches of protein samples. They are numbered from 1 to 7. Following further analysis using peptide digestions and MS by ESI-Q-TOF, the protein spots were identified as follows: 1 , heme oxygenase $1 ; 2$, aldose 1-epimerase family protein; 3, oxygen-evolving complex-related; $4,17.8 \mathrm{kDa}$ class I heat shock protein; 5, glycine-rich RNA-binding protein (GRP7), BetVI allergen family protein, and nascent polypeptide-associated protein; 6 , aspartyl protease family protein; and 7, expressed protein with unknown function. The protein features were summarized in Table 1. One of them (spot 3) exhibits experimental molecular mass smaller than the theoretically predicted. It might represent the proteolyzed fragments of the precursor form. For spot 5, three proteins were detected, suggesting that the level(s) of one, 


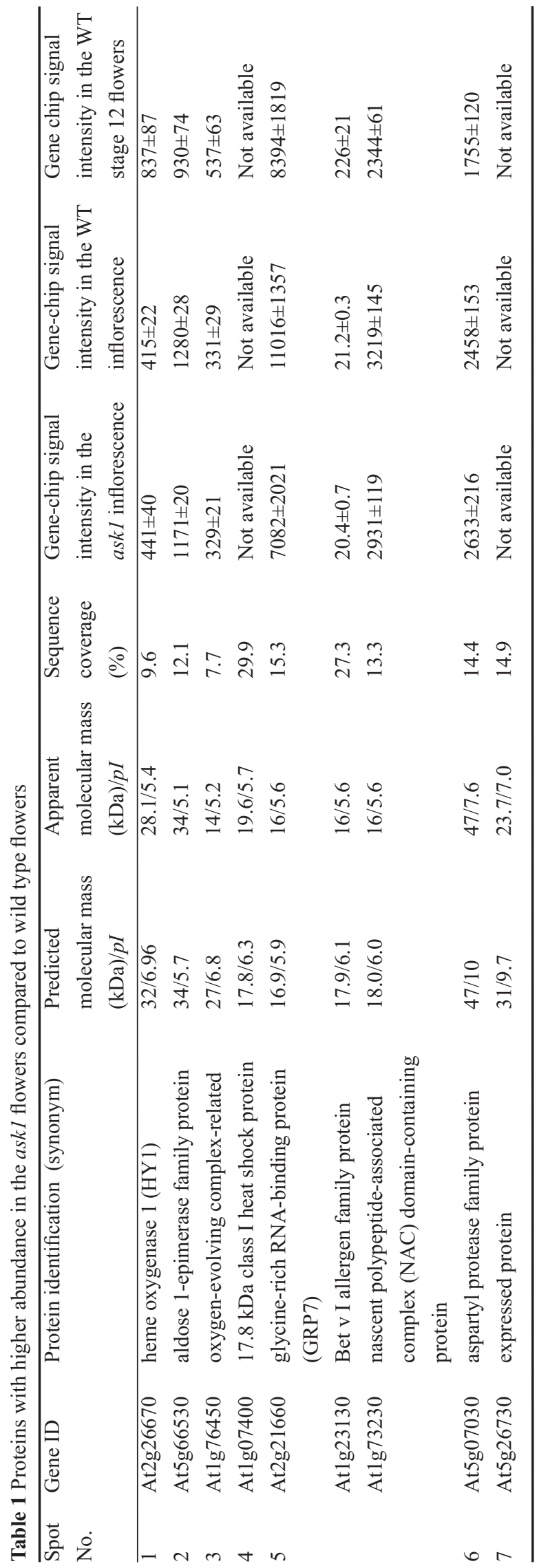

two or all of the three proteins could be affected by the ask1 mutation; all three proteins have a good score and match the observed $p I$ and $\mathrm{MW}$. The technical limitation of 2-DE in the range of $\mathrm{pH} 3-10$ also makes the extremely alkaline proteins difficult to focus in IPG strips (Bio-Rad 2-DE technical manual), thus appearing to have a lower $p I$ value than the theoretically predicted value, such as spot 6 and 7 . The protein spots identified can be classified into several types based on their predicted physiological functions. They are proteins related to phytochrome-mediated responses, circadian oscillations, post-translation modification and stress responses, which is consistent with the observation that ASK1 is involved in multiple physiological processes through interacting with different F-box proteins.

Proteins involved in phytochrome-mediated responses and circadian oscillations The proteomic approach identified one protein that is involved in phytochrome-mediated responses, HY1 (heme oxygenase, spot 1 in Figure2). HY1 catalyzes the conversion of heme to biliverdin (a precursor of phytochromobilin), thus playing an important role in red/ far-red light perception [24]. Our results indicate that HY1 is normally present at lower level in the developing flower and that ASK1 function is necessary for this reduced level. Because HY1 is a key enzyme for the production of the precursor of phytochromobilin, its up-regulation in the ask1 plants might make the perception of environmental light signals abnormal. Although phytochromes play important roles in regulating flowering, it is possible that a reduction of phytochrome function is needed for normal flower development. This idea is supported by the observation that a $h y 1$ mutation was able to suppress the floral reversion of flowers to shoots in an ag mutant [25], indicating that the loss of $H Y 1$ function promotes flower development. Simon et al. [26] reported that the HY1 gene negatively regulates CONSTANS $(\mathrm{CO})$. The $\mathrm{CO}$ function is required for the long-day dependent promotion of flowering [27]. The high levels of HY 1 protein in the ask 1 mutant flowers may result in lower levels of $C O$; it is possible that $C O$ is also important for normal flower development after the flowering transition, as supported by a positive role of $C O$ in controlling the floral meristem determinacy [28].

Spot 5 that was detected at a higher level in the askl mutant flower than in the wild type corresponds to three proteins: a glycine-rich RNA-binding protein (GRP7: At2g21660); Bet v I allergen family protein (At1g23130) and a nascent polypeptide-associated complex (NAC) domain-containing protein (At1g73230) (Table 1). All three proteins have a matching score that is more than 50 , and they all have similar predicted masses and $p I$ s. It is possible that one or two or all three of the proteins are accumulated in the ask1 mutant, although GRP7 has the closest predicted 
mass and $p I$. The microarray data showed that the mRNA of At1g23130 is below the detection limit in the young inflorescences. It is expressed at very low level in the stage 12 flowers, while the mRNA level of At1g73230 (nascent polypeptide-associated complex (AtNAC) domain-containing protein) is much higher than that of Atlg23130, and the level of of At2g21660 (AtGRP7) mRNA is the highest among three proteins (Table 1). AtGRP7 has been characterized as a component of a negative-feedback loop through which the circadian oscillations of its own transcript is regulated. The AtGPR7 protein accumulates with a certain delay and represses further accumulation of its transcript, presumably at the post-transcriptional level [29]. Microarray data suggest that, in the ask1 mutant, the AtGRP7 transcript level declines moderately compared to the wild type (table 1), consistent with the negative feedback loop. The F-box proteins (ZTL, FKF, LKP2) were demonstrated to regulate the circadian clock and cooperate with ASK1 in the regulation of circadian oscillations [17, 18]. It is possible that ASK1 participates in this negative feedback loop by targeting AtGRP7 for degradation, and the accumulation of AtGRP7 protein in the ask1 mutant cause the down-regulation of its own transcript. For other two proteins there is no reported functional characterization about them in plants. The nascent polypeptide-associated complex (NAC) domain-containing protein (At1g73230) is similar to a human RNA polymerase B transcription factor 3 and its mRNA level is very similar in all tissues (flowers, leaves, stems and roots) based on the gene chip microarray data [23]. It is likely a housekeeping gene.

Proteins related to post-translation modification An aspartyl protease (At5g07030) was identified to accumulate at a higher than normal level in the askl mutant. Some aspartyl proteases can cleave signal peptides within hydrophobic domains, and therefore, were named signal peptide peptidase (SPP) [30]. Intramembrane-cleaving proteases have become important regulators of cell growth, signaling, and pattern formation during development [31]. An aspartyl protease called PCS1 (At5g02190) in Arabidopsis was shown to be essential for early embryogenesis, and important but not essential for female and male gametogenesis. Knock-out of the aspartyl protease gene causes excessive cell death in reproductive organs and over-expression causes anther dehiscence failure [32]. There are several aspartyl protease genes expressed predominantly in Arabidopsis flower organs and their functions could be partly redundant in some of the reproductive tissues [32]. Therefore, aspartyl protease in flowers may act as an important tool to remove or activate a downstream molecule in the reproductive developmental processes. The abnormal high accumulation of the aspartyl protease in the askl mutant is consistent with the previous report that the $A S K 1$ and $A S K 2$ genes play a vital role in Arabidopsis embryogenesis [19], which may function by affecting the levels of the proteins essential for embryogenesis.

Stress inducible proteins We have also identified two stressinducible proteins that are increased in the askl mutant flowers. One of them, a $17.8 \mathrm{kDa}$ class I heat shock protein, is a typical stress induced protein that confers cytoprotection against oxidative injury. It could play an important protective role in maintaining normal development when ASK1 gene is disrupted. Another protein, an oxygen-evolving complex-related protein, encoded by At $1 \mathrm{~g} 76450$, is also increased in the ask1 mutant. Mutation of ASK1 gene may directly or indirectly cause an oxidative stress in flower organs but this still remains to be investigated further. This is not surprising because several signaling pathways are altered in the ask1 mutant, including responses to auxin $[11,12]$, GA [21], and jasmonic acid [11-14]. Therefore, the
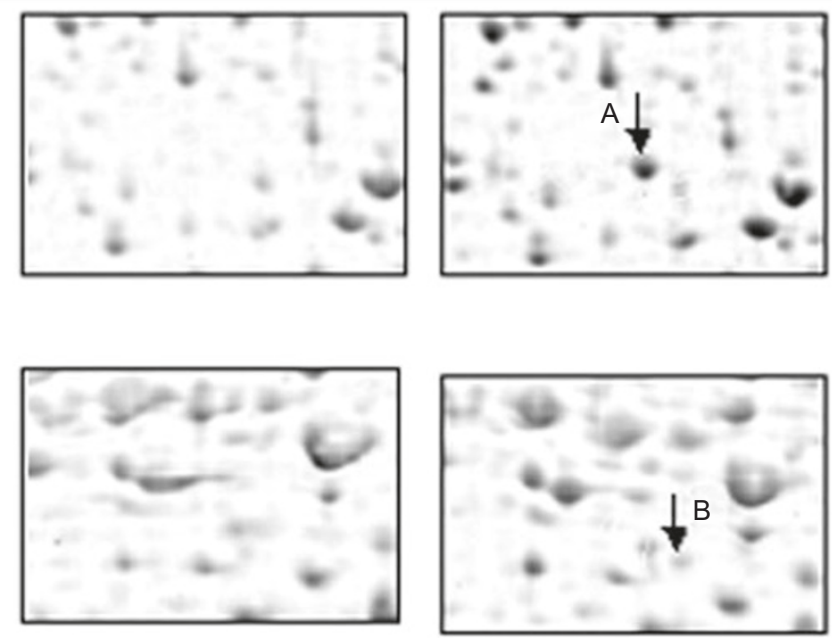

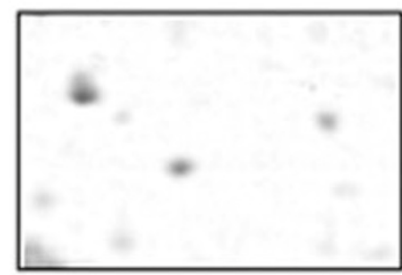

ask1

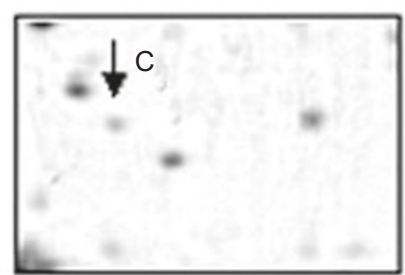

WT
Figure 3 Proteins with higher abundance in the wild type flowers compared to the askl mutant ones. The enlarged images are spot images after digitalization and spot matching. The proteins with different abundance are marked with arrows and numbered. A, xyloglucan endotransglycosylase; B, SC35-like splicing factor SCL30a; C, carbonic anhydrase; 
ask1 mutant may be abnormal in some stress responses.

\section{Proteins with decreased levels in the ask1 mutant}

Although the initial goal of this study is to identify proteins that have increased abundance in the askl mutant, we also found several proteins that are dramatically decreased in the mutant (Figure 3). Since it may also help to elucidate the roles of ASK1 protein plays in vivo, we discuss them in this paper.

Dramatic decrease of two proteins associated with hormone responses during floral development were observed in the ask1 mutant. They are XTH3 protein that belongs to xyloglucan endotransglycosylase (XET) family and SCL30a protein. In our experiments, the protein spot of XTH3 had an obvious decrease in the ask1 mutant flowers and could be easily identified each time. XET genes are predicted to encode cell wall enzymes involved in the construction and modification of the cellulose/xyloglucan framework, which is required for the normal organ growth. The transcript of $X T H 3$ is induced by IAA [33]. The microarray data also suggests that $X T H 3$ gene is specifically expressed at late stages of floral development (Table 2), indicating that XTH3 protein is probably important for IAA induced floral organ elongation at later stages, which is consistent with a reduced auxin signaling in the askl mutant. Another member of the XTH family, TCH4, is capable of modifying cell wall xyloglucans [34]. This protein is dramatically up regulated in response to the growth hormones auxin and brassinosteroids [34, 35]. Furthermore, the TCH4 expression was restricted to expanding tissues and organs that undergo cell wall modification, especially at the site of future bulge formation [36]. Akamatsu et al. [37] reported a mutant named acl5 that has a severe defect in the process of cell elongation after transition to the reproductive stage. RNA blotting showed that the $a c 15$ mutation causes a striking reduction in the transcript level of an XET (EXGT-A1) [38]. The ask1 mutant phenotypes of shorter stature, reduced number and size of petal and stamen filament length are consistent with the functions of the ASK1 protein in promoting cell expansion and division [1], which is realized by the expression of proteins responsible for the cell expansion and division. The down regulation of XTH3 protein in the ask1 mutant may result from the abnormal hormone signaling in the askl mutant flowers. From the early inflorescence (floral stage 1-9) to the stage-12 flower, the transcription level of XTH3 increased 100-fold in the wild type Arabidopsis (from 32 to 3713). This strongly implies that XET not only affects root hair bulge formation, but also is important for normal floral development.

SCL30a is a component of spliceosomal machinery that is responsible for the processing of nuclear RNA [39, 40].

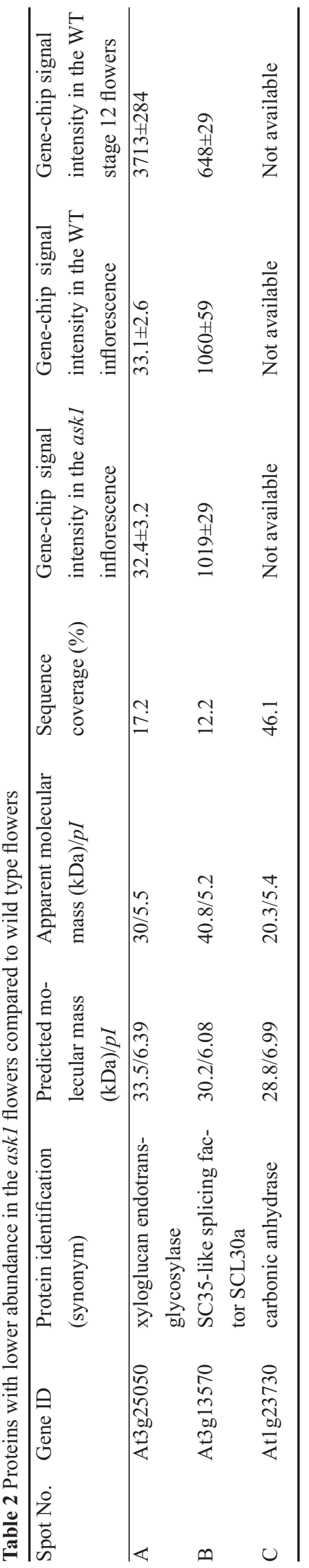


Using AtSCL30a as the bait in a yeast two-hybrid screen, Lopato et al. [40] found that it interacts with AtRSZ33, which is a phosphoprotein that concentrates in nuclear speckles and is predominantly present in roots and flowers. Furthermore, increased AtRSZ33 expression promotes cell expansion and changes polarization of cell elongation and division [39], suggesting that the levels of SCL30a may be important for regulating cell expansion during flower development. This is also consistent with the previous observation of Zhao et al. [2] that ask1-1 mutant has reduced growth of flower organs. ASK1 may regulate cell expansion by modulating the levels of proteins such as SCL30a, although likely indirectly.

This paper is the first proteomic analysis of the ask $1 \mathrm{mu}-$ tant. It is of interest to note that although the protein levels of the identified proteins showed very strikingly difference in the 2-D gel, the mRNA level did not show obvious difference at early stages for most of the proteins. Considering that the targets of ASK1-containing SCF complexes may have very important functions in floral organ identity, it is more important to study the early stages of flowers than the late stages. The non-correlation of protein levels and mRNA levels indicates the need to analyze protein levels, and not simply draw conclusions based on mRNA levels. Our results strongly demonstrate that the protein level can be regulated separately from the mRNA level and it is important to study protein level.

ASK1 can potentially regulate the level of a protein (call it X) in at least three different ways: (1) regulates the degradation of the protein X; (2) regulates the level and/or activity of a transcription factor (like AP3) responsible for the mRNA level of the protein X, but does not affect the degradation of $\mathrm{X}$; (3) change the development of the flower, such that the number of cells that express protein $\mathrm{X}$ is increased or decreased. Among the above possibilities, number 2 and 3 predict a correlation between mRNA and protein levels, barring more complex regulations. In contrast, for the first possibility, the mRNA levels might be similar between wild type and the askl mutant, but the protein levels would be different. In our experiments, six out of seven protein spots (if the other two proteins are excluded for spot5) with higher abundance in the ask1 mutant have very similar mRNA levels, indicating that the levels of these proteins do not reflect their mRNA levels. Therefore, these proteins are likely degraded by an ASK1-dependent proteolysis pathway, and thus are good candidate targets of ASK1-containing SCF complexes. For AtGRP7, the higher mRNA level in the wild type than in the ask 1 mutant is consistent with the previous report that it is a component of a negative-feedback loop through which the circadian oscillations of its own transcript is regulated [29]; so it can also be a candidate for the ASK1-containing
SCF complex.

In Arabidopsis, 694 potential F-box genes have been identified [20]. At least 18 out of the 21 Arabidopsis ASK genes were expressed under normal growth condition $[8,9]$. Among the $A S K$ genes, $A S K 1$ may play a major role in most fundamental processes regulated by SCF complexes, and ASK1 interacts with many F-box proteins in a yeast-two hybrid assay $[7,9,19,20]$. Therefore, numerous biological processes regulated by SCF complexes may be abnormal in the ask 1 mutant. In the meantime, the relative weak phenotypes in the ask 1 mutant compared to loss of function mutation in other core SCF component AtCUL1 and Rbx 1 make the ask1 mutant a unique material to study potential SCF complex target proteins involved in later stages of plant development such as floral development $[41,42]$. The weak ask 1 phenotype also has an advantage for proteomic analysis in that the protein difference between the ask1 mutant and wild type are not due to major tissue structure change, which is confirmed by the similar RNA level between the mutant and wild type. The proteomic analysis in this study provides an important initial view of the proteins affected by the $A S K 1$ gene in the floral development process of Arabidopsis. The identified proteins could be the protein targets for ASK1-mediated proteolysis pathway. For the down-regulated proteins in the askl mutant flowers, such as xyloglucan endotransglycosylase, it is of interest to determine its function in floral development. For those proteins with elevated levels in the askl mutant flowers, further investigations are needed to ascertain whether they were directly or indirectly regulated by ASK1; in other words, whether they are degraded through the ubiquitinmediated proteolysis pathway with ubiquitination by an ASK1-containing SCF complex.

\section{Acknowledgements}

We thank Dr Yue Jun from Institute of Genetics at Fudan University for kind help and advice on 2-DE technique, Hasan Koc from the proteomic center at the Pennsylvania State University for help with protein identification with MS and Qing Zhang for assistance with the normalization of the microarray data. This work was supported by the Youth Exploration Funding of School of Life Sciences at Fudan University and in part by a grant to H.M. from the US National Science Foundation (MCB-0092075).

\section{References}

1 Yang M, Hu Y, Lodhi M, McCombie WR, Ma H. The Arabidopsis SKP1-LIKE1 gene is essential for male meiosis and may control homologue separation. Proc Natl Acad Sci U S A 1999; 96:11416-11421.

2 Zhao D, Yang M, Solava J, Ma H. The ASK1 gene regulates 
development and interacts with the $U F O$ gene to control floral organ identity in Arabidopsis. Dev Genet 1999; 25:209-223.

3 Zhao D, Yu Q, Chen M, Ma H. The ASK1 gene regulates $\mathrm{B}$ function gene expression in cooperation with $U F O$ and $L E A F Y$ in Arabidopsis. Development 2001; 128:2735-2746.

4 Zhang H, Kobayashi R, Galaktionov K, Beach D. p19Skp1 and p45Skp2 are essential elements of the cyclin A-CDK2 S phase kinase. Cell 1995; 82:915-925.

5 Bai C, Sen P, Hofmann K, et al. SKP1 connects cell cycle regulators to the ubiquitin proteolysis machinery through a novel motif, the F-box. Cell 1996; 86:263-274.

6 Connelly C, Hieter P. Budding yeast $S K P 1$ encodes an evolutionarily conserved kinetochore protein required for cell cycle progression. Cell 1996; 86:275-285.

7 Zheng N, Schulman BA, Song L, et al. Structure of the Cul1Rbx1-Skp1-F boxSkp2 SCF ubiquitin ligase complex. Nature 2002; 416:703-709.

8 Zhao D, Ni W, Feng B, et al. Members of the ArabidopsisSKP1-like gene family exhibit a variety of expression patterns and may play diverse roles in Arabidopsis. Plant Physiol 2003; 133:203-217.

9 Farras R, Ferrando A, Jasik J, et al. SKP1-SnRK protein kinase interactions mediate proteasomal binding of a plant SCF ubiquitin ligase. Embo J 2001; 20:2742-2756.

10 Samach A, Klenz JE, Kohalmi SE, et al. The UNUSUAL FLORAL ORGANS gene of Arabidopsis thaliana is an F-box protein required for normal patterning and growth in the floral meristem. Plant J 1999; 20:433-445.

11 Ruegger M, Dewey E, Gray WM, et al. The TIR1 protein of Arabidopsis functions in auxin response and is related to human SKP2 and yeast grr1p. Genes Dev 1998; 12:198-207.

12 Gray WM, Kepinski S, Rouse D, Leyser O, Estelle M. Auxin regulates SCF(TIR1)-dependent degradation of AUX/IAA proteins. Nature 2001; 414:271-276.

13 Xie DX, Feys BF, James S, Nieto-Rostro M, Turner JG. COI1: an Arabidopsis gene required for jasmonate-regulated defense and fertility. Science 1998; 280:1091-1094.

$14 \mathrm{Xu}$ L, Liu F, Lechner E, et al. The SCF(COI1) ubiquitin-ligase complexes are required for jasmonate response in Arabidopsis. Plant Cell 2002; 14:1919-1935.

15 Dieterle M, Zhou YC, Schafer E, Funk M, Kretsch T. EID1, an F-box protein involved in phytochrome A-specific light signaling. Genes Dev 2001; 15:939-944.

16 Schwechheimer C, Serino G, Deng XW. Multiple ubiquitin ligase-mediated processes require COP9 signalosome and AXR1 function. Plant Cell 2002; 14:2553-2563.

17 Somers DE, Schultz TF, Milnamow M, Kay SA. ZEITLUPE encodes a novel clock-associated PAS protein from Arabidopsis. Cell 2000; 101:319-329.

18 Nelson DC, Lasswell J, Rogg LE, Cohen MA, Bartel B. FKF1, a clock-controlled gene that regulates the transition to flowering in Arabidopsis. Cell 2000; 101:331-340.

19 Liu F, Ni W, Griffith ME, et al. The ASK1 and ASK2 genes are essential for Arabidopsis early development. Plant Cell 2004; 16:5-20.

20 Gagne JM, Downes BP, Shiu SH, Durski AM, Vierstra RD. The F-box subunit of the SCF E3 complex is encoded by a diverse superfamily of genes in Arabidopsis. Proc Natl Acad Sci U S A 2002; 99:11519-11524.
21 Sasaki A, Itoh H, Gomi K, et al. Accumulation of phosphorylated repressor for gibberellin signaling in an F-box mutant. Science 2003; 299:1896-1898.

22 Bradford MM. A rapid and sensitive method for the quantitation of microgram quantities of protein utilizing the principle of protein-dye binding. Anal Biochem 1976; 72:248-254.

23 Zhang X, Feng B, Zhang Q, et al. Genome-wide expression profiling and identification of gene activities during early flower development in Arabidopsis. Plant Mol Biol 2005; 58:401419.

24 Schepens I, Duek P, Fankhauser C. Phytochrome-mediated light signalling in Arabidopsis. Curr Opin Plant Biol 2004; 7:564569.

25 Okamuro JK, den Boer BG, Lotys-Prass C, Szeto W, Jofuku KD. Flowers into shoots: photo and hormonal control of a meristem identity switch in Arabidopsis. Proc Natl Acad Sci U S A 1996; 93:13831-13836.

26 Simon R, Igeno MI, Coupland G. Activation of floral meristem identity genes in Arabidopsis. Nature 1996; 384:59-62.

27 Putterill J, Robson F, Lee K, Simon R, Coupland G. The CONSTANS gene of Arabidopsis promotes flowering and encodes a protein showing similarities to zinc finger transcription factors. Cell 1995; 80:847-857.

28 Mizukami Y, Ma H. Determination of Arabidopsis floral meristem identity by AGAMOUS. Plant Cell 1997; 9:393-408.

29 Heintzen C, Nater M, Apel K, Staiger D. AtGRP7, a nuclear RNA-binding protein as a component of a circadian-regulated negative feedback loop in Arabidopsis thaliana. Proc Natl Acad Sci U S A 1997; 94:8515-8520.

30 Urny J, Hermans-Borgmeyer I, Gercken G, Schaller HC. Expression of the presenilin-like signal peptide peptidase (SPP) in mouse adult brain and during development. Gene Expr Patterns 2003; 3:685-691.

31 Selkoe D, Kopan R. Notch and Presenilin: regulated intramembrane proteolysis links development and degeneration. Annu Rev Neurosci 2003; 26:565-597.

32 Ge X, Dietrich C, Matsuno M, et al. An Arabidopsis aspartic protease functions as an anti-cell-death component in reproduction and embryogenesis. EMBO Rep 2005; 6:282-288.

33 Yokoyama R, Nishitani K. A comprehensive expression analysis of all members of a gene family encoding cell-wall enzymes allowed us to predict cis-regulatory regions involved in cell-wall construction in specific organs of Arabidopsis. Plant Cell Physiol 2001; 42:1025-1033.

$34 \mathrm{Xu}$ W, Purugganan MM, Polisensky DH, et al. Arabidopsis $\mathrm{TCH} 4$, regulated by hormones and the environment, encodes a xyloglucan endotransglycosylase. Plant Cell 1995; 7:15551567.

35 Rose JK, Braam J, Fry SC, Nishitani K. The XTH family of enzymes involved in xyloglucan endotransglucosylation and endohydrolysis: current perspectives and a new unifying nomenclature. Plant Cell Physiol 2002; 43:1421-1435.

36 Vissenberg K, Martinez-Vilchez IM, Verbelen JP, Miller JG, Fry SC. In vivo colocalization of xyloglucan endotransglycosylase activity and its donor substrate in the elongation zone of Arabidopsis roots. Plant Cell 2000; 12:1229-1237.

37 Akamatsu T, Hanzawa Y, Ohtake Y, et al. Expression of endoxyloglucan transferase genes in acaulis mutants of Arabidopsis. Plant Physiol 1999; 121:715-722. 
38 Hanzawa Y, Takahashi T, Komeda Y. ACL5: an Arabidopsis gene required for internodal elongation after flowering. Plant J 1997; 12:863-874.

39 Kalyna M, Lopato S, Barta A. Ectopic expression of atRSZ33 reveals its function in splicing and causes pleiotropic changes in development. Mol Biol Cell 2003; 14:3565-3577.

40 Lopato S, Forstner C, Kalyna M, et al. Network of interactions of a novel plant-specific Arg/Ser-rich protein, atRSZ33, with atSC35-like splicing factors. J Biol Chem 2002; 277:3998939998.
41 Shen WH, Parmentier Y, Hellmann H, et al. Null mutation of AtCUL1 causes arrest in early embryogenesis in Arabidopsis. Mol Biol Cell 2002; 13:1916-1928.

42 Lechner E, Xie D, Grava S, et al. The AtRbx1 protein is part of plant SCF complexes, and its down-regulation causes severe growth and developmental defects. J Biol Chem 2002; 277:50069-50080.

Edited by Hong Wei Xue 